\title{
SINGULAR BEHAVIOR IN NONLINEAR PARABOLIC EQUATIONS
}

\author{
BY \\ WEI - MING NI ${ }^{1}$ AND PAUL SACKS ${ }^{2}$
}

\begin{abstract}
In this paper, we study the well-posedness of the initial-boundary value problems of some quasilinear parabolic equations, namely, nonlinear heat equations and the porous medium equation in the fast-diffusion case. We establish nonuniqueness (local in time) and/or nonregularizing effect of these equations in some critical cases. The key which leads to the resolution of these problems is to study some singular solutions of the elliptic counterparts of these parabolic problems (the so-called $M$-solutions of the Lane-Emden equations in astrophysics).
\end{abstract}

Introduction. In this article we are concerned first of all with the existence or nonexistence of singular solutions of certain semilinear elliptic boundary value problems, and secondly with the application of these results to the study of some related parabolic problems.

To begin with, consider the problem

$$
\begin{aligned}
\Delta u+f(u) & =0, & & x \in \Omega, \\
u & =0, & & x \in \partial \Omega, \\
u & >0, & & x \in \Omega,
\end{aligned}
$$

where $\Omega=$ unit ball in $\mathbf{R}^{N}, N \geqslant 3, \Delta=\sum_{i=1}^{N}\left(\partial^{2} / \partial x_{i}^{2}\right)$ and $f \in C^{1}\left(\overline{\mathbf{R}^{+}}\right)$. By a singular solution of (0.1) we mean a function $u \in C^{2}(\Omega \backslash\{0\}), \mathrm{u}>0$, in $\Omega, \mathrm{u}=0$ for $x \in \partial \Omega$ and $\overline{\lim }_{x \rightarrow 0} u(x)=\infty$.

Equations of the type (0.1) arise in a variety of physical problems; for instance, in astrophysics, equation (0.1) with $f(u)=u^{p}$ is known as the Lane-Emden equation. See Chandrasekhar [10], Joseph and Lundgren [17], and Ni [23]. Singular solutions have intrinsic interest in some of these problems, and have not been investigated as thoroughly as classical solutions.

We denote by $\lambda_{1}=\lambda(\Omega)$ the first eigenvalue of $-\Delta$ in $\Omega$ with zero boundary conditions.

TheOREM 1. (i) Suppose

$$
0<f(s)<s f^{\prime}(s) \leqslant \frac{N}{N-2} f(s), \quad \dot{s}>0,
$$

Received by the editors March 29, 1984.

1980 Mathematics Subject Classification. Primary 35J60, 35K55, 35R25.

${ }^{1}$ Supported in part by NSF grant MCS 82-00033.

${ }^{2}$ Supported in part by Iowa State University Science and Humanities Research Foundation and by Office of Naval Research Contract \# N00014-83-K-0051. 


$$
f^{\prime}(0)<\lambda_{1} \text {. }
$$

Then (0.1) has infinitely many singular solutions.

(ii) Suppose

$$
0<\frac{N+2}{N-2} f(s)<s f^{\prime}(s), \quad s>0 .
$$

Then 0.1 has no singular solutions.

REMARK. At the expense of additional technical complications in the proof, one can allow equality in the right-hand of inequality $(0.4)$. However, we shall not prove this here.

If we are interested in the case of the exact power law $f(s)=s^{p}, p>1$, then $(0.2)$, (0.3) includes the case $p \in(1, N /(N-2)]$ while inequality (0.4) includes $p \in$ $((N+2) /(N-2), \infty)$. Actually, if we are willing to make more specific assumptions on the nonlinearity $f$ we can include the remaining power law cases (for $p>1$ at least) as well as allowing some $x$ dependence.

Consider, for example, the following problem:

$$
\begin{aligned}
\Delta u+\lambda|x|^{l} u^{p} & =0, & & x \in \Omega, \\
u & =0, & & x \in \partial \Omega, \\
u & >0, & & x \in \Omega,
\end{aligned}
$$

where $\lambda>0, l \geqslant 0$ and $p>1$. This equation arises in the study of rotating stellar structures; see Hénon [16].

THEOREM 2. (i) If $1<p<(N+2+2 l) /(N-2)$, then (0.5) has infinitely many singular solutions.

(ii) If $p \geqslant(N+2+2 l) /(N-2)$, then (0.5) has no singular solution which is radially symmetric.

REMARKS. (i) If $l=0$ one may show by slight modification of the arguments in Gidas, Ni and Nirenberg [11] that any singular solution must be radially symmetric, hence we obtain the nonexistence of any singular solution in this case.

(ii) The nonexistence results in Theorems 1 and 2 correspond to a theorem of Pohozaev [30] for classical solutions. We do not make any direct use of Pohozaev's results in studying singular solutions.

(iii) Due to the homogeneity in the equation, the question of existence of solutions of (0.5) (singular or classical) may be studied via phase plane techniques. See e.g. Joseph and Lundgren [17], Chandrasekhar [10], and Aviles [2] for some special cases. However, the methods we use here will work also for more general nonlinearities as in Theorem 1. See also Lions [22] for some related results.

(iv) The exact behavior of singular solutions near $x=0$ has been investigated by various authors. See, for example, Serrin [31], Gilbarg and Serrin [14], Lions [22], Gidas and Spruck [13], and Aviles [2].

(v) For the type of nonlinearities $f$ considered in Theorems 1 and 2 it turns out always that singular solutions exist exactly when classical solutions exist. Now this is not the case for general $f$. Indeed in the case $f(u)=\lambda u, \lambda \geqslant 0$, it is not hard to see 
that there cannot exist both classical and singular solutions of $(0.1)$, although one or the other may exist, depending on the value of $\lambda$.

We are also interested in the following two parabolic problems:

$$
\begin{aligned}
& u_{t}=\Delta u+u^{p}, \quad x \in \Omega, t>0, \\
& u(x, t)=0, \quad x \in \partial \Omega, t>0, \\
& u(x, 0)=u_{0}(x), \quad x \in \Omega, t=0 ; \\
& u_{t}=\Delta\left(u^{m}\right), \quad x \in \Omega, t>0, \\
& u(x, t)=0, \quad x \in \partial \Omega, t>0, \\
& u(x, 0)=u_{0}(x), \quad x \in \Omega .
\end{aligned}
$$

Consider first the semilinear problem (0.6).. The theory of this problem set in $L^{q}$ spaces has been developed by Weissler [33] and others. In particular, it is known that if $u_{0} \in L^{q}(\Omega), q>N / 2(p-1)$ and $q \geqslant p$ then (0.6) has a unique solution from $C\left([0, T] ; L^{q}(\Omega)\right)$, for some $T>0$. In the marginal case $p=q=N(p-1) / 2=$ $N /(N-2)$ one still has a local existence theorem; we show uniqueness fails in this case.

THEOREM 3. If $p=N /(N-2), N \geqslant 3, \Omega=$ ball in $\mathbf{R}^{N}$, then there exists (infinitely many) $u_{0} \in L^{p}(\Omega)$ such that $(0.6)$ has at least two solutions from $C\left([0, T] ; L^{p}(\Omega)\right)$, for some $T>0$.

REMARKS. (i) The existence theorems mentioned above may be found in Weissler [33]. The uniqueness when $q>N(p-1) / 2$ and $q \geqslant p$ is proven by a more or less standard contraction mapping argument, using the regularizing properties of the semigroup generated by $-\Delta$. See Baras [3] for details.

(ii) Some related nonuniqueness results have been proved in Haraux and Weissler [15] and Baras [3]. The exponent $p=N /(N-2)$ is also a limiting case in the work of Baras.

(iii) The nonlinearity $u^{p}$ in Theorem 3 could be replaced by $f(u)$, provided $f$ satisfies $(0.2),(0.3)$,

$$
\varliminf_{s \rightarrow \infty} \frac{f(s)}{s^{p}}>0, \quad p=\frac{N}{N-2}
$$

and

$$
f^{\prime}(s) \leqslant C_{1} s^{p-1}+C_{2}, \quad s>0 .
$$

We will explain why these hypotheses are sufficient following the proof of Theorem 3.

Next we turn to the problem (0.6) with $p \geqslant(N+2) /(N-2)$. It is well known that solutions of this problem may blow up in finite time (Kaplan [18], Levine [21]). On the other hand, any solution which is uniformly bounded for $t \geqslant 0$ tends to zero as $t \rightarrow \infty$. Indeed, if $t_{n} \rightarrow \infty$ then there exists a subsequence $t_{n_{k}} \rightarrow \infty$ such that $u\left(\cdot, t_{n_{k}}\right)$ converges to a nonnegative steady state solution of (0.6). However, by the theorem of Pohozaev [30] the only such solution is the zero solution. 
in the paper [28] it is shown that there also exist solutions of $(0.6)$ which exist for all $t \geqslant 0$ but are not uniformly bounded. We are interested in what can be said about the asymptotic behavior of these solutions.

THEOREM 4. Let $u$ be a nonnegative global classical solution of (0.6) with $p \geqslant$ $(N+2) /(N-2), N \geqslant 3$, and $u_{0}(x)=u_{0}(|x|)$. Then

$$
\begin{gathered}
\lim _{t \rightarrow \infty} u(x, t)=0, \quad x \neq 0, \\
\lim _{t \rightarrow \infty}\|u(\cdot, t)\|_{L^{1}(\Omega)}=0 .
\end{gathered}
$$

The conclusions $(0.10)$ and $(0.11)$ remain valid for a global weak solution $u$ provided

$$
u\left(\cdot, t_{0}\right) \in L^{\infty}(\Omega) \text { for some } t_{0} \geqslant 0
$$

and

(0.13) $\quad u$ is a monotone increasing limit of classical solutions of (0.6).

REMARKS. (i) Here by a weak solution we mean a function $u \in C\left([0, T] ; L^{1}(\Omega)\right) \cap$ $L^{p}(\Omega \times(0, T))$ for any $T>0$ satisfying

$$
\int_{s}^{\tau} \int_{\Omega}\left(u \zeta_{t}+u \Delta \zeta+u^{p} \zeta\right) d x d t=\left.\int_{\Omega} u(\cdot, t) \zeta(\cdot, t) d x\right|_{s} ^{\tau}
$$

for any $0 \leqslant s \leqslant \tau \leqslant T<\infty$ and $\zeta \in C^{2}(\bar{\Omega} \times[0, T])$ satisfying $\zeta(x, t)=0$ for $x \in$ $\partial \Omega$.

(i) In [28] it is the existence of weak global unbounded solutions which is demonstrated. The conditions $(0.12)$ and $(0.13)$ are satisfied by the construction given there. It would, of course, be interesting to know whether these solutions are classical or not; we suspect that they are, but have not been able to prove this.

(iii) Again, exact homogeneity in the nonlinearity is not necessary. The conclusion of Theorem 4 remains valid with $u^{p}$ replaced by $f(u)$ where $f$ satisfies $(0.3),(0.4)$, and $f$ is convex.

Finally, consider the "fast diffusion" problem $(0.7)$ with $m \in(0,1)$ (see e.g. Berryman and Holland [6] or Peletier [29].) It is known from Véron [32] or Bénilan [4] that if $m>(N-2 q) / N$ and $u_{0} \in L^{q}(\Omega), q \geqslant 1$, then $u(\cdot, t) \in L^{\infty}(\Omega)$ for all $t>0$. For $q=1$ the cut-off exponent has recently been shown to be sharp. Namely, it follows from the results in Brézis and Friedman [7] that if $m \leqslant(N-2) / N$ and $t_{0}>0$ there exists $u_{0} \in L^{1}(\Omega)$ such that $u\left(\cdot, t_{0}\right) \notin L^{\infty}(\Omega)$.

Using Theorem 2 and looking for separable solutions of $(0.7)$ we obtain further counterexamples to this regularizing effect.

THEOREM 5. Let $q \in[1,2 N /(N+2)), m \in((N-2) /(N+2),(N-2 q) / N)$ and $t_{0}>0$. Then there exists a solution of $(0.7)$ with $u_{0} \in L^{q}(\Omega)$ and $u\left(\cdot, t_{0}\right) \notin L^{\infty}(\Omega)$.

If $q=1$ we may allow $m=(N-2) / N$.

REMARK. By the use of comparison arguments one sees that if there is no $L^{\infty}$ regularizing effect in the case that $\Omega$ is a ball in $\mathbf{R}^{N}$, then there cannot be regularizing for any other domain. 
The organization of this paper is as follows. $\$ 1$ contains some auxiliary results related to problems $(0.1)$ and $(0.5)$. In $\S 2$ we give the proofs of Theorems 1 and 2. Finally, in $\$ 3$ we give the proofs of Theorems ?, 4 and 5.

1. In this section we prove or recall some preliminary results.

LEMMA 1.1. Let $u \in C^{2}(\Omega \backslash\{0\})$ be a nonnegative function satisfying

$$
\Delta u+f(x, u)=0, \quad x \in \Omega \backslash\{0\},
$$

with $f \geqslant 0, f \in C^{1}(\bar{\Omega} \times[0, \infty))$. Then:

(i) $f(x, u(x)) \in L^{1}(\Omega)$.

(ii) If $|x|^{m} u^{q}(x) \in L^{1}(\Omega)$ for some $q \geqslant(N+m) /(N-2)$, then

$$
\Delta u+f(x, u)=0 \text { in } \mathscr{D}^{\prime}(\Omega) .
$$

COROLlaRy 1.2. Let $u \in C^{2}(\Omega \backslash\{0\})$ be a nonnegative function satisfying

$$
\Delta u+f(x, u)=0, \quad x \in \Omega \backslash\{0\},
$$

with $f \geqslant 0, f \in C^{1}(\bar{\Omega} \times[0, \infty))$ and

$$
\lim _{s \rightarrow \infty} \inf _{x \in \Omega} \frac{f(x, s)}{|x|^{m} s^{q}}>0 \text { for some } q \geqslant \frac{N+m}{N-2} .
$$

Then

$$
\Delta u+f(x, u)=0 \quad \text { in } \mathscr{D}^{\prime}(\Omega) .
$$

REMARKs. (i) Lemma 1.1 is a slight generalization of Proposition 3.1 in Gidas, Ni and Nirenberg [12]. For the reader's convenience we sketch the proof here. The result of part (i) is also contained in Theorem 1 of Brézis and Lions [8].

(ii) The conclusion of the corollary is false in general if (1.3) does not hold. For example, if $f(x, u)=|x|^{l} u^{p}, 1<p<(N+l) /(N-2)$, then it may be shown as in Lions [22] that any singular solution of (1.1) must satisfy

$$
\Delta u+|x|^{l} u^{p}=\alpha \delta_{0} \quad \text { in } \mathscr{D}^{\prime}(\Omega)
$$

for some $\alpha<0$, where $\delta_{0}$ denotes the Dirac mass at $x=0$.

Proof of Lemma 1.1. (i) As in Brézis and Lions [8] we choose cut-off functions $\zeta_{\varepsilon}(x)$ in the form

$$
\zeta_{\varepsilon}(x)=\Phi\left(\varepsilon /|x|^{N-2}\right)
$$

where $\Phi \in C^{\infty}\left(\mathbf{R}^{+}\right)$is convex, $\Phi(0)=1$ and $\Phi(t) \equiv 0$ for $t \geqslant 1$. Then $\zeta_{\varepsilon}(x)=0$ for $|x| \leqslant \varepsilon, 0 \leqslant \zeta_{\varepsilon}(x) \leqslant 1, \zeta_{\varepsilon}(x) \rightarrow 1$ as $\varepsilon \rightarrow 0$ for $x \neq 0$ and $\Delta \zeta_{\varepsilon} \geqslant 0$ in $\Omega$. Multiplication of the equation by $\zeta_{\varepsilon}$ and integration over $\Omega$ yields

$$
\int_{\Omega} f(x, u(x)) \zeta_{\varepsilon}(x) d x \leqslant \int_{|x|=1}\left(u \zeta_{\varepsilon r}-\zeta_{\varepsilon} u_{r}\right) d r
$$

The right-hand side is bounded independently of $\varepsilon$, hence we obtain the conclusion from Fatou's lemma.

(ii) Choose $\tau \in C^{\infty}(0, \infty), \tau(r) \equiv 0$ for $0 \leqslant r \leqslant 1, \tau(r) \equiv 1$ for $r \geqslant 2,0 \leqslant \tau \leqslant 1$ and $\tau_{\varepsilon}(r)=\tau(r / \varepsilon)$. It suffices to show that

$$
\lim _{\varepsilon \rightarrow 0} \int_{\Omega} \tau_{\varepsilon}(u \Delta \zeta+f(x, u) \zeta) d x=0
$$

for any $\zeta \in C_{0}^{\infty}(\Omega)$; note that $u \in L^{1}(\Omega)$ under the stated hypotheses. 
Integrating by parts and using the equation for $u$, one sees that it is enough to have

$$
\lim _{\varepsilon \rightarrow 0} \int_{\Omega} \zeta u \Delta \tau_{\varepsilon} d x=0
$$

and

$$
\lim _{\varepsilon \rightarrow 0} \int u \nabla \zeta \cdot \nabla \tau_{\varepsilon} d x=0
$$

We may write $u(x)=h^{1 / q}(x) /|x|^{m / q}$ with $h \in L^{1}(\Omega)$ and $q \geqslant(N+m) /(N-2)$. Since $\left|\Delta \tau_{\varepsilon}\right| \leqslant C \varepsilon^{-2}$ we obtain the following estimate for the integral in (1.5) using Hölder's inequality;

$$
\begin{aligned}
\left|\int_{\Omega} u \zeta \Delta \tau_{\varepsilon} d x\right| & \leqslant \frac{C}{\varepsilon^{2}} \int_{|x|<2 \varepsilon} \frac{h^{1 / q}}{|x|^{m / q}} d x \\
& \leqslant \frac{C}{\varepsilon^{2}}\left(\int_{|x|<2 \varepsilon} h d x\right)^{1 / q}\left(\int_{|x|<2 \varepsilon} \frac{d x}{|x|^{m q^{\prime} / q}}\right)^{1 / q^{\prime}}, \frac{1}{q}+\frac{1}{q^{\prime}}=1, \\
& \leqslant C\left(\int_{|x|<2 \varepsilon} h d x\right)^{1 / q} \varepsilon^{N(1-1 / q)-m / q-2}
\end{aligned}
$$

and the right-hand side tends to zero with $\varepsilon$ by virtue of the assumptions made. The proof of (1.6) is similar.

LEMMA 1.3. Let $u \in C^{2}(\Omega \backslash\{0\})$ be a nonnegative radially symmetric function satisfying

$$
\Delta u+\lambda|x|^{l} u^{p}=0, \quad x \in \Omega \backslash\{0\},
$$

with $\lambda>0, l \geqslant 0$ and $p>1$. Then

$$
u(x) \leqslant C /|x|^{(l+2) /(p-1)}, \quad x \in \Omega \backslash\{0\} .
$$

REMARK. The estimate (1.7) is not sharp in all cases; see [2, 8, 22]. We will need this lemma only for $p \in((N+l) /(N-2),(N+2+2 l) /(N-2))$, in which case (1.7) is proved in Gidas and Spruck [13] where no assumption of radial symmetry is made. As the proofs there are somewhat complicated, we prefer to give a simple derivation for the case at hand.

Proof. Assume first that $p \in(1,(N+2+2 l) /(N-2)]$. Let $v(r)=$ $r^{(l+2) /(p-1)} u(r)$; we need to show that $v \in L^{\infty}(0,1)$. Making the further change of variable $w(s)=v\left(s^{1 /(2-N)}\right)$ we need $w \in L^{\infty}(1, \infty)$. The equation for $w$ is

$$
w^{\prime \prime}+\frac{2 \mu}{s} w^{\prime}+\frac{w}{s^{2}}\left(\mu(\mu-1)+\frac{\lambda}{(N-2)^{2}} w^{p-1}\right)=0,
$$

where $\mu=(l+2) /((p-1)(N-2)) \geqslant \frac{1}{2}$.

We first claim that if $w$ is monotonically increasing on $\left(s_{0}, \infty\right)$ for some $s_{0} \geqslant 1$ then

$$
\lim _{s \rightarrow \infty} w(s) \leqslant\left(\frac{N-2}{2 \lambda^{2}}\right)^{2 /(p-1)} .
$$


Otherwise, we would have $(\lambda /(N-2)) w^{p-1}(s)>\frac{1}{4}$ for some $s_{1} \geqslant s_{0}$, and we derive a contradiction using a Sturm comparison type argument. Indeed, for $\sigma>\frac{1}{4}$ solutions of

$$
h^{\prime \prime}+2 \mu h^{\prime} / s+(\mu(\mu-1)+\sigma) h / s^{2}=0
$$

are oscillatory, hence a solution of (1.8) cannot have constant sign on $\left(s_{1}, \infty\right)$.

We deduce that if $w$ is not bounded on $(1, \infty)$ there must exist a sequence $t_{n} \rightarrow \infty$ such that $w^{\prime}\left(t_{n}\right)=0$ and $w\left(t_{n}\right) \rightarrow \infty$. To see that this is impossible also, we multiply the equation (1.8) by $s^{2} w^{\prime}$ and integrate from 1 to $t_{n}$. Integration by parts yields

$$
(2 \mu-1) \int_{1}^{t_{n}} s\left(w^{\prime}\right)^{2} d s+\left.\left[\frac{\mu(\mu-1) w^{2}}{2}+\frac{\lambda}{(p+1)(N-2)^{2}} w^{p+1}\right]\right|_{1} ^{t_{n}}=\frac{w^{\prime}(1)^{2}}{2} \text {. }
$$

In particular, since $\mu>\frac{1}{2}$,

$$
\frac{\mu(\mu-1) w^{2}\left(t_{n}\right)}{2}+\frac{\lambda w^{p+1}\left(t_{n}\right)}{(p+1)(N-2)^{2}} \leqslant \text { constant, }
$$

which is inconsistent with $w\left(t_{n}\right) \rightarrow \infty$.

If $p \in((N+2+2 l) /(N-2), \infty)$ the arguments are similar except that one makes the change of variable $w(s)=v\left(s^{\gamma}\right)$ where

$$
1 / \gamma=2-N+2((l+2) /(p-1)) \text {. }
$$

Another important ingredient in the proofs of Theorems 1 and 2 is the following uniqueness result. Denote by $\Omega_{R}$ the annulus $\left\{x \in \mathbf{R}^{N}: R<|x|<1\right\}$.

TheOREM 1.4. (i) Let $p>1, l \geqslant 0$ and $\lambda>0$. Then the problem (0.5) has at most one radially symmetric classical solution.

(ii) The same is true if $\Omega$ is replaced by $\Omega_{R}, R>0$.

(iii) Assume (0.2) holds. Then the problem (0.1) has at most one classical solution.

(iv) Assume (0.2) holds. Then the problem (0.1) in $\Omega_{R}, R>0$, has at most one radially symmetric classical solution.

REMARKS. (i) Parts (ii), (iii), (iv) here have been proved in Ni and Nussbaum [26], with some special cases proved previously in $\mathrm{Ni}$ [25]. The proof of part (i) is by a simple scaling argument, as in Gidas, Ni and Nirenberg, p. 224 [11]. In part (iii) no assumption of radial symmetry was necessary since this is a necessary consequence of Theorem 1 of [11]; similarly in part (i) this need not be assumed if $l=0$. In the case of the annulus there may exist nonradial as well as radial solutions; cf. Brézis and Nirenberg [9].

2. In this section we give the proofs of Theorems 1 and 2. The techniques used here are closely related to those used in $\mathrm{Ni}$ [25], and $\mathrm{Ni}$ and Nussbaum [26].

We begin with the existence assertions. We look for singular solutions which are radially symmetric, $u(x)=u(r), r=|x|$. Letting $s=r^{2-N}, v(s)=u(r)$, the equation and side conditions to be satisfied are

$$
\begin{aligned}
& v^{\prime \prime}+g(s, v)=0, \quad s>1, \\
& v(1)=0, \quad \lim _{s \rightarrow \infty} v(s)=\infty,
\end{aligned}
$$


where

$$
g(s, v)=f(v) /(N-2)^{2} s^{2+2 /(N-2)}
$$

in the case of Theorem 1, and

$$
g(s, v)=\lambda v^{p} /(N-2)^{2} s^{2+(l+2) /(N-2)}
$$

in the case of Theorem 2.

Consider the associated initial value problem

$$
\begin{aligned}
& w^{\prime \prime}+g(s, w)=0, \quad s>1, \\
& w(1)=0, \quad w^{\prime}(1)=\alpha
\end{aligned}
$$

with $\alpha \in(0, \infty)$ and denote the solution by $w_{\alpha}$ insofar as it exists.

Under the hypotheses of part (i) of either Theorem 1 or 2 , the problem $(0.1)$ or (0.5) has exactly one radially symmetric classical solution. The existence of this solution for (0.1), or (0.5) in case $l=0$ follows, for example, from Ambrosetti and Rabinowitz [1]; one then has radial symmetry from Theorem 1 of Gidas, $\mathrm{Ni}$ and Nirenberg [11] and uniqueness of radial solutions from Theorem 1.4. The problem (0.5) with $l>0$ is investigated in $\mathrm{Ni}$ [24] where it is shown that there exists a radially symmetric solution from $H_{0}^{1}(\Omega)$. Furthermore, it is also shown in [24] that if $u \in H_{0}^{1}(\Omega)$ and is radially symmetric then

$$
|u(r)| \leqslant C\|u\|_{H_{0}^{1}(\Omega)} r^{1-N / 2} .
$$

It follows that $|x|^{l} u^{p-1} \in L^{q}(\Omega)$ for some $q>N / 2$, whence $u$ must be a classical solution of $(0.5)$, by elliptic regularity theory [20]. Uniqueness within the radial class again follows from Theorem 1.4.

Observe next that if $\alpha$ is such that $\lim _{s \rightarrow \infty} w_{\alpha}(s)=L$ for some $L \in[0, \infty)$ then reversing the earlier change of variables we obtain a solution $u$ of $(0.1)$ or $(0.5)$ in $\Omega \backslash\{0\}$ with $u \in L^{\infty}(\Omega)$. By Lemma 1.1 and elliptic regularity arguments again, $u$ must be a classical solution in $\Omega$. We conclude that under the stated hypotheses there exists exactly one number $\alpha_{0}>0$ such that $\lim _{s \rightarrow \infty} w_{\alpha_{0}}=L$ for some $L \in(0, \infty)$.

Now consider

$$
T(\alpha)=\sup \left\{\bar{s}: w_{\alpha}(s)>0 \text { on }(1, \bar{s})\right\}
$$

and

$$
I=\text { domain of } T=\{\alpha \in(0, \infty): T(\alpha)<\infty\} .
$$

From considerations of continuous dependence it is not hard to see that $I$ is open, $T$ is continuous on $I$, and $T$ is unbounded on any component of $I$. On the other hand, $T$ is one-to-one by Theorem 1.4 , and $I \neq(0, \infty)$ since $\alpha_{0} \notin I$. It follows that the complement of $I$ contains an interval. Since clearly either $T(\alpha)<\infty$ or else $\lim _{s \rightarrow \infty} w_{\alpha}(s)=\infty, \alpha \neq \alpha_{0}$, we obtain the existence of a continuum of singular solutions.

REMARK. In Ni [25] or Ni and Nussbaum [26] it is actually shown that $T(\alpha)$ is monotone decreasing. Thus it is not hard to see that $I=(\beta, \infty)$ for some $\beta>0$.

We turn now to the proof of part (ii) of Theorem 1. As remarked earlier, any singular solution is necessarily radially symmetric. 
Set $\theta=\frac{1}{2}(N+2) /(N-2), \gamma=2 \theta$ and $H(t)=t^{-\gamma} f(t) /(N-2)^{2}$; note that the condition (0.4) implies that $H^{\prime}(t)>0$ on $(0, \infty)$. The equation satisfied by $z(s)=$ $s^{-1 / 2} u\left(s^{1 /(2-N)}\right)$ may be written

$$
\left(s z^{\prime}\right)^{\prime}+\frac{z}{s}\left(z^{\gamma-1} H(\sqrt{s z})-\frac{1}{4}\right)=0, \quad s>1 .
$$

It is sufficient to show that if $z(1)=0$ and $z^{\prime}(1)>0$, then $z(T)=0$ for some $T \in(1, \infty)$.

We claim first that $z$ cannot be monotone nondecreasing on $(1, \infty)$. To see this, suppose $z$ is increasing on $(1, \infty)$ and let $L=\lim _{s \rightarrow \infty} z(s)$. If both $L$ and $H_{\infty} \equiv$ $\lim _{t \rightarrow \infty} H(t)$ are finite, then clearly we must have $L^{\gamma-1} H_{\infty}>\frac{1}{4}$ since otherwise $s z^{\prime}(s) \geqslant z^{\prime}(1)>0$ which contradicts $L<\infty$. Thus, in any case, there must exist $s_{1}>1$ and $\varepsilon>0$ such that

$$
z^{\gamma-1}(s) H(\sqrt{s} z(s))-\frac{1}{4} \geqslant \varepsilon
$$

for $s \geqslant s_{1}$.

Letting $h(s)$ denote the solution of

$$
\left(s h^{\prime}\right)^{\prime}+\varepsilon h / s=0, \quad h\left(s_{1}\right)=z\left(s_{1}\right), \quad h^{\prime}\left(s_{1}\right)=z^{\prime}\left(s_{1}\right),
$$

$h(s)$ is oscillatory, and by an oscillation comparison argument it follows that $z$ must change sign on $\left(s_{1}, \infty\right)$, a contradiction.

Next we claim that $z^{\prime}$ cannot have an accumulation point of zeros (this is immediate if $f$ is analytic). To see this, set

$$
g(s, z)=\frac{z}{s}\left(z^{\gamma-1} H(\sqrt{s} z)-\frac{1}{4}\right) .
$$

If it were true that $z^{\prime}$ had an accumulation point of zeros at a point $s=a$ with $z(a)=L>0$, it would follow that

$$
\left.\frac{\partial^{k}}{\partial s^{k}} g(s, z)\right|_{\substack{s=a \\ z=L}}=0
$$

for every positive integer $k$. However, using (2.3) with $k=0$ and $k=1$ and doing some fairly straightforward manipulations, one obtains $t f^{\prime}(t)=\gamma f(t)$ for $t=\sqrt{a L}$, contradicting (0.4).

Next we claim that $z$ cannot have a positive local minimum in $(1, \infty)$. Otherwise, by the previous remark there is a first such local minimum at $s=\xi$, and we may find $\eta \in(1, \xi)$ such that $z(\eta)=z(\xi), z(s)>z(\xi)$ for $s \in(\eta, \xi)$. Also there exists $\zeta \in$ $(\eta, \xi)$ such that $z$ is increasing on $(\eta, \zeta)$ and decreasing on $(\zeta, \xi)$. Multiplying the equation (2.2) by $s z^{\prime}$ and integrating from $\eta$ to $\xi$ yields

$$
\int_{\eta}^{\xi} z^{\gamma} H(\sqrt{s} z) z^{\prime} d s \geqslant 0
$$

To derive a contradiction we write this integral as the sum of the integrals from $\eta$ to $\zeta$ and $\zeta$ to $\xi$. On each of these two intervals we make the change of variable $\sigma=z(s)$. Let $a=z(\eta)=z(\xi), b=z(\zeta)$ and $s_{1}(\sigma)$ and $s_{2}(\sigma)$ be the functions inverse to $z$ with 
ranges in $(\eta, \zeta)$ and $(\zeta, \xi)$, respectively. Then (2.4) may be written

$$
0 \leqslant \int_{a}^{b} \sigma^{\gamma}\left(H\left(\sqrt{s_{1}(\sigma)} \sigma\right)-H\left(\sqrt{s_{2}(\sigma)} \sigma\right)\right) d \sigma .
$$

Since $H$ is strictly increasing and $s_{2}(\sigma)>s_{1}(\sigma)$ for $\sigma \in(a, b)$, this is a contradiction.

If then $z(s)>0$ on $(1, \infty)$ the only remaining possibility is that there exists $s_{0} \in(1, \infty)$ such that $z$ is increasing on $\left(1, s_{0}\right)$, decreasing on $\left(s_{0}, \infty\right)$, and $\lim _{s \rightarrow \infty} z(s)=L$ for some $L \geqslant 0$. Note that we cannot have $\lim _{s \rightarrow \infty}\left|s z^{\prime}(s)\right|>0$ since otherwise $z(s) \rightarrow-\infty$ as $s \rightarrow \infty$. There must therefore exist sequences $\xi_{m} \rightarrow \infty$ and $\eta_{m}, \eta_{m+1}<\eta_{m}<s_{0}<\xi_{m}<\xi_{m+1}$ such that $\xi_{m} z^{\prime}\left(\xi_{m}\right) \rightarrow 0, z\left(\eta_{m}\right)=z\left(\xi_{m}\right), z(s)$ $>z\left(\xi_{m}\right)$ for $s \in\left(\eta_{m}, \xi_{m}\right)$.

Again multiplying the equation by $s z^{\prime}$ and integrating from $\eta_{m}$ to $\xi_{m}$ we get

$$
\left.\frac{\left(s z^{\prime}\right)^{2}}{2}\right|_{\eta_{m}} ^{\xi_{m}}+\int_{\eta_{m}}^{\xi_{m}} z^{\gamma} H(\sqrt{s} z) z^{\prime} d s=0
$$

Changing variables as before gives

$$
\int_{b_{m}}^{a} \sigma^{\gamma}\left(H\left(\sqrt{s_{1}(\sigma)} \sigma\right)-H\left(\sqrt{s_{2}(\sigma)} \sigma\right)\right) d \sigma \geqslant-\frac{\left(\xi_{m} z^{\prime}\left(\xi_{m}\right)\right)^{2}}{2},
$$

where $b_{m}=z\left(\xi_{m}\right)$. Letting $m \rightarrow \infty$ we obtain the nonnegativity of the left-hand integral with lower limit replaced by $L$, which is again a contradiction. This completes the proof of Theorem 1.

The proof of Theorem 2(ii) in the case $p>(N+2+2 l) /(N-2)$ is similar except slightly simpler. The critical case $p=(N+2+2+l) /(N-2)$ must be handled slightly differently; it is done essentially in $\mathrm{Ni}$ [23, Appendix]. We refer the interested reader to this source.

3. In this section we give the proofs of Theorems 3, 4, and 5 .

Proof of Theorem 3. It follows from Theorem 1 of Weissler [33] that for any $u_{0} \in L^{p}(\Omega), p=N /(N-2)$, the problem $(0.6)$ has a solution

$$
u \in C\left([0, T] ; L^{p}(\Omega)\right) \cap L^{\infty}((\tau, T) \times \Omega)
$$

for some $T>0$ and any $\tau \in(0, T)$.

On the other hand, if we take $l=0$ we obtain from Theorem 2 infinitely many singular solutions of the steady state equation (0.5), each of which also satisfies $(0.5)$ in $\mathscr{D}^{\prime}(\Omega)$ by Corollary 1.2. Since by Lemma 1.1 these solutions belong to $L^{p}(\Omega)$, they are then also solutions of (0.6) from $C\left([0, T] ; L^{p}(\Omega)\right)$ for any $T>0$ and clearly must be different from the solution given by Weissler's theorem.

REMARK. As mentioned earlier, the nonlinearity $u^{p}$ may be replaced by $f(u)$, satisfying (0.2), (0.3), (0.8) and (0.9). Indeed, due to (0.9) we may obtain from Theorem 3 of Weissler [33] a solution of (0.6) which belongs to $L^{\infty}(\Omega)$ for $t>0$; (0.2) and (0.3) guarantee the existence of an unbounded steady state singular solution by Theorem 1; and, finally, singular solutions are distributional solutions by Corollary 1.2 since $(0.8)$ holds. 
Proof of TheOrem 4. Let $u$ be a nonnegative global classical solution of (0.6) with $p \geqslant(N+2) /(N-2), N \geqslant 3$, and $u_{0}(x)=u_{0}(|x|)$. By Theorem 1 of $\mathrm{Ni}$ and Sacks [27] there exists $T^{*} \geqslant 0$ depending on $u_{0}$ such that $u_{r}(r, t) \leqslant 0$ for $t \geqslant T^{*}$ and $r \in[0,1]$. Thus replacing $u(x, t)$ by $u\left(x, t+T^{*}\right)$ if necessary, we may suppose that $u(\cdot, t)$ is radially decreasing for every $t \geqslant 0$.

Let $\lambda_{1}$ and $\psi_{1}$ denote the first eigenvalue and eigenfunction of $-\Delta$ in $\Omega$ with zero boundary conditions; then $\psi_{1} \geqslant 0$ and we may take $\int_{\Omega} \psi_{1} d x=1$. Multiplying the equation by $\psi_{1}$ and integrating gives

$$
\begin{aligned}
\frac{d}{d t} \int u \psi_{1} d x & =\int_{\Omega} u^{p} \psi_{1} d x-\lambda_{1} \int_{\Omega} u \psi_{1} d x \\
& \geqslant\left(\int u \psi_{1} d x\right)^{p}-\lambda_{1} \int u_{j 1} d x .
\end{aligned}
$$

Thus, since $u$ exists for all $t \geqslant 0$ by hypothesis we must have

$$
\int_{\Omega} u(\cdot, t) \psi_{1} d x \leqslant \lambda_{1}^{1 /(p-1)}
$$

for all $t \geqslant 0$. Moreover, it is clear that there exists a constant $C=C(\Omega)$ such that

$$
\int_{\Omega} h d x \leqslant C \int_{\Omega} h \psi_{1} d x
$$

if $h$ is any radially decreasing function on $\Omega$. Thus we have

$$
\|u(\cdot, t)\|_{L^{1}(\Omega)} \leqslant C_{0}
$$

for all $t \geqslant 0$ for some $C_{0}<\infty$ depending only on $\Omega$ and $p$.

Next if $|x|=R>0$ then

$$
u(x, t) \leqslant \frac{C}{R^{N}} \int_{|x|<R} u(y, t) d y \leqslant \frac{C}{R^{N}} \int_{\Omega} u(y, t) d y \leqslant \frac{C_{0}}{R^{N}},
$$

using again the fact that $u(\cdot, t)$ is radially decreasing. In particular,

$$
u \in L^{\infty}\left((0, \infty) \times \Omega_{R}\right)
$$

for any $R>0$ (recall $\Omega_{R}=\left\{x \in \mathbf{R}^{N}: R \geqslant|x|<1\right\}$ ).

Using parabolic regularity theory [19] and the fact that $u$ is uniformly bounded on $(0, \infty) \times \Omega_{R / 2}$ we obtain a bound for $u, u_{t}, u_{x_{i}}, u_{x_{i} x_{j}}$ as in $C^{\alpha, \alpha / 2}\left((0, \infty) \times \bar{\Omega}_{R}\right)$ for any $R>0$ and $\alpha \in(0,1)$. Here $C^{\alpha, \alpha / 2}$ denotes the space of Hölder continuous functions with exponent $\alpha$ in $x$ and $\alpha / 2$ in $t$.

We claim now that $u_{t}(\cdot, t) \rightarrow 0$ in $L^{2}\left(\Omega_{R}\right)$ as $t \rightarrow \infty$, for any $R>0$. To see this, multiply the equation by $u_{t}$ and integrate to obtain

$$
\int_{0}^{t} \int_{\Omega} u_{t}^{2} d x d t+J(u(\cdot, t))=J\left(u_{0}\right)
$$

where

$$
J(v)=\frac{1}{2} \int|\nabla v|^{2} d x-\frac{1}{p+1} \int v^{p+1} d x
$$


The fact that $u$ is globally defined implies that $J(u(\cdot, t)) \geqslant 0$ for all $t \geqslant 0$; see e.g. Levine [21]. Therefore

$$
\int_{0}^{\infty} \int_{\Omega_{R}} u_{t}^{2} d x d t \leqslant C<\infty
$$

Since $t \rightarrow \int_{\Omega_{R}} u_{t}^{2} d x$ is uniformly continuous, by the uniform Hölder estimate on $u_{t}$, we must have

$$
\lim _{t \rightarrow \infty} \int_{\Omega_{R}} u_{t}^{2}(\cdot, t) d x=0 .
$$

To prove $(0.10)$ it is enough to show that if $t_{n} \rightarrow \infty$ there is a subsequence $t_{n_{k}} \rightarrow \infty$ such that $u\left(x, t_{n_{k}}\right) \rightarrow 0, x \neq 0$.

Let $t_{n} \rightarrow \infty$. First of all $\left\{u\left(\cdot, t_{n}\right)\right\}$ is uniformly bounded in $C^{2+\alpha}\left(\bar{\Omega}_{R}\right)$ for any fixed $R>0$ and $\alpha \in(0,1)$. Thus we may find a subsequence $t_{n_{k}} \rightarrow \infty$ and $w \in$ $C^{2}\left(\bar{\Omega}_{R}\right)$ such that $u\left(\cdot, t_{n_{k}}\right) \rightarrow w$ in $C^{2}\left(\bar{\Omega}_{R}\right)$. Furthermore, since $u_{t}\left(\cdot, t_{n_{k}}\right) \rightarrow 0$ in $L^{2}\left(\Omega_{R}\right)$ we must have

$$
\Delta w+w^{p}=0, \quad x \in \Omega_{R} .
$$

By the use of a diagonalization argument we may find a further subsequence, again denoted $t_{n_{k}}$, and $w \in C^{2}(\Omega \backslash\{0\})$ such that

$$
\Delta w+w^{p}=0, \quad x \in \Omega \backslash\{0\},
$$

and $u\left(x, t_{n_{k}}\right) \rightarrow w(x)$ for $x \neq 0$. Clearly $w$ satisfies the boundary condition $w(x)=0$ for $x \in \partial \Omega$.

If $w \notin L^{\infty}(\Omega)$ then $w$ is a radially symmetric nonnegative singular solution of (0.5), and this contradicts Theorem 2. Therefore we must have $w \in L^{\infty}(\Omega)$. By Lemma 1.1 and elliptic regularity [20], $w$ must, in fact, be a classical solution of (0.5). But then Pohozaev's theorem [30] implies $w \equiv 0$. This completes the proof of $(0.10)$.

Next we prove $(0.11)$; suppose to the contrary that there exists $t_{n}$ such that

$$
\left\|u\left(\cdot, t_{n}\right)\right\|_{L^{1}(\Omega)} \geqslant \varepsilon>0 .
$$

By (3.4) it follows that

$$
\int_{\Omega} u\left(\cdot, t_{n}\right) \psi_{1}, d x \geqslant \bar{\varepsilon}=\frac{\varepsilon}{C}>0 .
$$

Now from (3.2) we obtain easily that

$$
\frac{d}{d t} \int_{\Omega} u \psi_{1} \geqslant-\lambda_{1}\left(\frac{\lambda_{1}}{p}\right)^{1 /(p-1)}\left(1-\frac{1}{p}\right),
$$

and so thare exists $\delta>0$ such that for $s \in\left(t_{n}, t_{n}+\delta\right)$

$$
\int_{\Omega} u(\cdot, s) \psi_{1} d x \geqslant \frac{\bar{\varepsilon}}{2} \text {. }
$$

Next, integrating (3.1) from $t_{n}$ to $t_{n}+\delta$ gives

$$
\begin{aligned}
\int_{t_{n}}^{t_{n}+\delta} \int_{\Omega} u^{p}(\cdot, t) \psi_{1}, d x d t & \leqslant \int_{\Omega} u\left(\cdot, t_{n}+\delta\right) \psi_{1} d x+\lambda_{1} \int_{t_{n}}^{t_{n}+\delta} \int_{\Omega} u(\cdot, t) \psi_{1} d x d t \\
& \leqslant \lambda_{1}^{1 /(p-1)}\left(1+\lambda_{1} \delta\right),
\end{aligned}
$$


using (3.3). By the mean value theorem there must exist $s_{n} \in\left(t_{n}+\delta\right)$ such that

$$
\int_{\Omega} u^{p}\left(\cdot, s_{n}\right) \psi_{1} d x \leqslant \frac{\lambda_{1}^{1 / p-1)}\left(1+\lambda_{1} \delta\right)}{\delta} .
$$

We have therefore found a sequence $s_{n} \rightarrow \infty$ such that $u\left(\cdot, s_{n}\right) \psi_{1} \rightarrow 0$ pointwise a.e. and $\left\|u\left(\cdot, s_{n}\right) \psi_{1}\right\|_{L^{p}(\Omega)}$ is uniformly bounded. It follows immediately that $u\left(\cdot, s_{n}\right) \psi_{1} \rightarrow 0$ in $L^{1}(\Omega)$, which conflicts with (3.16).

This completes the proof of Theorem 4 in the case that $u$ is classical. If, instead, $u$ is a weak solution satisfying $(0.12)$ and $(0.13)$ it is only necessary to make some of the above arguments more carefully, and to use approximation at appropriate points.

Suppose $u_{n}(x, t) \uparrow u(x, t)$ for each $(x, t)$ where each $u_{n}$ is a global classical solution of (0.6). By (0.13) we may assume $u_{0} \in C_{0}^{1}(\bar{\Omega})$. From the proof of Theorem 1 in [27] it follows that there exists $T^{*}<\infty$ independent of $n$ such that $u_{n}(\cdot, t)$ is radially decreasing for $t \geqslant T^{*}$; hence the same is true of $u$. The estimates (3.3) and (3.5) remain valid for $u$ by approximation (alternatively these inequalities can be derived directly for weak solutions). We have (3.9) holding with $u$ replaced by $u_{n}$ and constant $C$ independent of $n$; by lower semicontinuity (3.9) is valid for $u$ itself. For the remainder of the proof of $(0.10)$ the arguments take place away from $x=0$, i.e. where the weak solution $u$ is actually classical.

In proving $(0.11)$ one has instead of $(3.15)$ the integrated form

$$
\int_{\Omega} u(\cdot, t) \psi_{1} d x-\int_{\Omega} u(\cdot, s) \psi_{1} d x \geqslant-\lambda_{1}\left(\frac{\lambda_{1}}{p}\right)^{1 /(p-1)}\left(1-\frac{1}{p}\right)(t-s),
$$

$t>s$, from which (3.16) again follows. The estimate (3.17) is still valid by the defintion of weak solution, and, instead of using the mean value theorem to derive (3.18), we have from (3.17)

$$
\operatorname{meas}\left\{s \in\left(t_{n}, t_{n}+\delta\right): \int u^{p}(\cdot, s) \psi_{1} d x \geqslant k\right\} \leqslant \frac{\lambda_{1}^{1 /(p-1)}\left(1+\lambda_{1} \delta\right)}{k},
$$

and the right-hand side is less than $\delta$ for $k>\lambda_{1}^{1 /(p-1)}\left(1+\lambda_{1} \delta\right) / \delta$. Clearly one then has (3.18) with a slightly larger constant on the right-hand side.

With these changes the proof of Theorem 4 for weak solutions is complete.

Proof of TheOREM 5. The problem (0.7) has solutions in the form

$$
u(x, t)=[C-(1-m) t]^{+1 /(1-m)} w^{p}(x)
$$

for $C>0, p=1 / m$ and where $w \in L^{p}(\Omega)$ satisfies $\Delta w+w^{p}=0$ in $\mathscr{D}^{\prime}(\Omega)$ and $w=0$ classically on $\partial \Omega$.

Since $p \in[N /(N-2),(N+2) /(N-2))$ we see from Theorem 2 and Corollary 1.2 that we can find such a function $w$ with $w \notin L^{\infty}(\Omega)$; choosing $C>(1-m) t_{0}$ then guarantees that $u\left(\cdot, t_{0}\right) \notin L^{\infty}(\Omega)$.

If $p=N /(N-2)$ then, from Lemma $1.1, u_{0}(x)=C^{1 /(1-m)} w^{p}(x)$ belongs to $L^{1}(\Omega)$. In the case that $p \in(N /(N-2),(N+2) /(N-2)), q>1$, it follows, from Lemma 1.3 and the fact that $u_{0}$ is smooth away from $x=0$, that $u_{0} \in L^{q}(\Omega)$ since $1 / p=m<(N-2 q) / N$. 
REMARK. In Gidas and Spruck [13] it is shown that $|x|^{2 / p-1)} u(x) \geqslant C>0$ if $u$ is a singular solution of $(0.5)$ with $l=0$ and $p \in(N /(N-2),(N+2) /(N-2))$. Using this fact, one may state the following more precise version of Theorem 5 . If $r \in(1,2 N /(N+2))$ and $m=(N-2 r) / N$, then the problem $(0.7)$ has a separable solution $u(x, t)$ such that $u_{0} \in L^{q}(\Omega)$ for any $q<r$ and $u(\cdot, t) \notin L^{r}(\Omega)$ for $t \in$ $[0, T]$, some $t>0$.

In the case $m=(N-2) / N$ then $(0.7)$ has a separable solution $u(x, t)$ such that $u_{0} \in L^{1}(\Omega)$ and $u(\cdot, t) \notin L^{q}(\Omega)$ for any $q>1$ on $[0, T]$ some $T>0$. To see this it only remains to check that the singular solutions of $(0.5)$ with $l=0$ and $p=$ $N /(N-2)$ do not belong to $L^{r}(\Omega)$ for any $r>p$. One argument for this is a bootstrap argument using elliptic $L^{p}$ (Calderón and Zygmund) estimates in a fairly standard way. Another approach is to use Theorem 1 of Aviles [2] where the exact behavior of $w$ near $x=0$ is obtained. We omit the details.

Since the above argument only works in the case $m=(N-2) / N$, we do not recover the nonregularizing theorem of Brézis and Friedman [7]. We conjecture that there should be some direct argument, perhaps along the lines of Bénilan and Diaz [5] by which lack of regularizing for some $m$ implies lack of regularizing for any smaller $m$.

\section{REFERENCES}

1. A. Ambrosetti and P. Rabinowitz, Dual variational methods in critical point theory and applications, J. Funct. Anal. 14 (1973), 349-381.

2. P. Aviles, On isolated singularities in some nonlinear partial differential equations, Indiana Univ. Math. J. 32 (1983), 773-791.

3. P. Baras, Non-unicité des solutions d' une equation d'evolution non-lineaire, preprint.

4. P. Bénilan, Opérateurs accrétifs et semigroups dans les espaces $L^{p}(1 \leqslant p \leqslant \infty)$, France-Japan Seminar, Tokyo, 1976.

5. P. Bénilan and J. I. Diaz, Comparison of solutions of nonlinear evolution problems with different nonlinear terms, Israel J. Math. 42 (1982), 241-257.

6. J. Berryman and C. Holland, Stability of the separable solution for fast diffusion, Arch. Rational Mech. Anal. 74 (1980), 379-388.

7. H. Brézis and A. Friedman, Nonlinear parabolic equations involving measures as initial conditions, J. Math. Pures Appl. (9) 62 (1983), 73-97.

8. H. Brézis and P. L. Lions, A note on isolated singularities for linear elliptic equations, Adv. in Math. Suppl. Stud., Vol. 7A, Academic Press, New York and London, 1981, pp. 263-266.

9. H. Brézis and L. Nirenberg, Positive solutions of nonlinear elliptic equations involving critical Sobolev exponents, Comm. Pure Appl. Math. 36 (1983), 437-477.

10. S. Chandrasekhar, An introduction to the study of stellar structures, Dover, New York, 1957.

11. B. Gidas, W.-M. Ni and L. Nirenberg, Symmetry and related properties via the maximum principle, Comm. Math. Phys. 68 (1979), 209-243.

12. Symmetry of positive solutions of nonlinear elliptic equations in $\mathbf{R}^{n}$, Adv. in Math. Suppl. Stud., Vol. 7A, Academic Press, New York and London, 1981, pp. 369-402.

13. B. Gidas and J. Spruck, Global and local behavior of positive solutions of nonlinear elliptic equations, Comm. Pure Appl. Math. 34 (1981), 525-598.

14. D. Gilbarg and J. Serrin, On isolated singularities of solutions of second order elliptic differential equations, J. Analyse Math. 4 (1955), 309-340.

15. A. Haraux and F. Weissler, Nonuniqueness for a similinear initial value problem, Indiana Univ. Math. J. 31 (1982), 167-189.

16. M. Hénon, Numerical experiments on the stability of spherical stellar systems, Astronom. and Astrophys. 24 (1973), 229-238.

17. D. Joseph and T. Lundgren, Quasilinear Dirichlet problems driven by positive sources, Arch. Rational Mech. Anal. 49 (1973), 241-269. 
18. S. Kaplan, On the growth of solutions of quasilinear parabolic equations, Comm. Pure Appl. Math. 16 (1963), 305-330.

19. O. A. Ladyzenskaja, V. A. Solonnikov and N. N. Ural'ceva, Linear and quasilinear equations of parabolic type, Amer. Math. Soc., Providence, R. I., 1968.

20. O. A. Ladyzenskaja and N. N. Ural'ceva, Linear and quasilinear equations of elliptic type, Academic Press, New York and London, 1968.

21. H. A. Levine, Some nonexistence and stability theorems for solutions of formally parabolic equations of the form $P u_{t}=-A u+F(u)$, Arch. Rational Mech. Anal. 5 (1973), 371-386.

22. P. L. Lions, Isolated singularities in semilinear problems, J. Differential Equations 38 (1980), 441-450.

23. W.-M. Ni, Uniqueness, nonuniqueness and related questions in nonlinear elliptic and parabolic equations, Proc. Sympos. Pure Math. (to appear).

24. $\ldots$ A nonlinear Dirichlet problem on the unit ball and its applications, Indiana Univ. Math. J. 31 (1982), 801-807.

25. _ Uniqueness of solutions of nonlinear Dirichlet problems, J. Differential Equations 50 (1983), 289-304.

26. W.-M. Ni and R. Nussbaum, Uniqueness and nonuniqueness for positive radial solutions of $\Delta u+$ $f(u, r)=0$, Comm. Pure Appl. Math. (to appear).

27. W.-M. Ni and P. Sacks, The number of peaks of positive solutions of semilinear parabolic equations, SIAM J. Math. Anal. (to appear).

28. W.-M. Ni, P. Sacks and J. Tavantzis, On the asymptotic behavior of solutions of certain quasilinear parabolic equations, J. Differential Equations 54 (1984), 97-120.

29. L. Peletier, The porous medium equation, Application of Nonlinear Analysis in the Physical Sciences, Pitman, New York, 1981.

30. S. Pohozaev, Eigenfunctions of the equation $\Delta u+\lambda f(u)=0$, Soviet Math. J. 6 (1965), 1408-1411.

31. J. Serrin, Local behavior of solutions of quasilinear equations, Acta Math. 111 (1964), 247-302.

32. L. Véron, Effets regularisants de semigroupes nonlinearies das des espaces de Banach, Ann. Fac. Sci. Toulouse Math. (5) 1 (1979), 171-200.

33. F. Weissler, Local existence and nonexistence for semilineàr parabolic equations in $L^{p}$, Indiana Univ. Math. J. 29 (1980), 79-102.

School of Mathematics, University of Minnesota, Minneapolis, Minnesota 55455

Department of Mathematics, Iowa State University, Ames, Iowa 50011 